\title{
The Politicization of Medical Preprints on Twitter during the Early Stages of COVID-19 Pandemic
}

\author{
ALEKSANDRA URMAN \\ STEFANIA IONESCU \\ University of Zurich, Switzerland \\ DAVID GARCIA \\ Graz University of Technology, Austria; Complexity Science Hub Vienna, \\ Austria; Medical University of Vienna, Austria

\section{ANIKÓ HANNÁK} \\ University of Zurich, Switzerland

\begin{abstract}
We examine the patterns of medical preprint sharing on Twitter during the early stages of the COVID-19 pandemic. Our analysis demonstrates a stark increase in attention to medical preprints among the general public since the beginning of the pandemic. We also observe a political divide in medical preprint sharing patterns - a finding in line with previous observations regarding the politicisation of COVID-19-related discussions. In addition, we find that the increase in attention to preprints from the members of the general public coincided with the change in the social media-based discourse around preprints.
\end{abstract}

Keywords: COVID-19, Twitter, preprints, political communication

\footnotetext{
$\square$ Aleksandra Urman: urman@ifi.uzh.ch Date submitted: 2021-08-19

Copyright @ 2022 (Urman A., Ionescu S., Garcia D., Hannak A.). Licensed under the Creative Commons Attribution-NonCommercial-NoDerivatives 4.0 International Public License. Available at: http://journalqd.org
} 
Preprints - versions of scholarly papers published ahead of peer review - are by now a form of publishing that is an integral part of the scientific ecosystem in many disciplines (Majumder and Mandl, 2020). In 1991, arXiv became the first open-access online repository of academic preprints, and by now it hosts over 1.8 million preprints in the fields of Physics, Mathematics, Computer Science, Quantitative Biology, Quantitative Finance, Statistics, Electrical Engineering and Systems Science, and Economics. Some disciplines like Computer Science or Physics were early adopters of preprint sharing while other fields, including life sciences, have started using this practice only recently (Berg et al., 2016). A dedicated preprint archive for medical research - medRxiv - was established as one of the latest such platforms in 2019. It became increasingly used in 2020 after the onset of the COVID-19 pandemic with the number of preprints published in medRxiv and bioRxiv (a preprint repository for biological sciences) surging since January 2020 (Homolak et al., 2020). During the early stages of the COVID-19 pandemic, scientists published more preprints than during any disease outbreak that happened before (Kupferschmidt, 2020).

At the same time, the discourse surrounding the COVID-19 pandemic in its early stages has been highly politicized in certain countries - for instance, in the US (Plohl and Musil, 2020). Under such circumstances, not-yet-peer-reviewed preliminary findings can be used as arguments in politicized discussions on medical issues and amplified online. If preliminary findings are later disconfirmed or proven to be not entirely correct, it is difficult to debunk them among the general public. That has to do with the continued influence effect and, in the case of politicized discussions, politically motivated cognition (van der Linden et al., 2021). Thus, in an environment where medical discussions are politicized, preprints can become a political tool and, in extreme cases, even contribute to the proliferation of medical misinformation online should their findings be disconfirmed. This highlights the necessity to examine the usage of medical preprints in the context of politicization - a question we address with the present study.

The rest of the paper is organized as follows: we begin by providing an overview of research regarding, first, the benefits and risks of preprints as a form of scholarly 
communication and, second, the politicization of medical information; then, we outline our research questions and describe our methodology; finally, we report and discuss the results.

\section{Preprints as a Form of Academic Publishing: Benefits and Risks}

Researchers acknowledge that preprints bring benefits such as the faster dissemination of findings, increased feedback opportunities, and broader access to scientific research (Chiarelli et al., 2019). In times of a global pandemic such as the COVID-19, scholars researching different aspects of the new disease have an incentive to share the results quickly, as it can help advance the understanding of the disease and potentially speed up the development of vaccines and other medical treatments as well as inform the development of prevention strategies. This is particularly valuable as, due to the rapid increase in the number of submissions, standard peer-review publication systems quickly get overloaded with new research on COVID-19 and cannot always process it in a timely manner (Homolak et al., 2020). In such extraordinary circumstances, preprints can be helpful as they facilitate a faster spread of new relevant scientific findings (Berg et al., 2016; Chiarelli et al., 2019; Homolak et al., 2020).

There is, however, one key issue associated with the sharing of preprints: since they are not subject to peer-review the findings reported in preprints can be flawed. This is especially true for medical preprints, as they do not require pre-registration of clinical trials. Thus, the presented results should be used with caution. Scientists typically take this into account and laboriously scrutinize the results of non-peer-reviewed research. However, scientists are not the only ones getting information from preprints; during the COVID-19 pandemic, the general public has been increasingly engaging with preprints

(Fraser et al., 2021). Since members of the general public might be less knowledgeable about the inner-workings of academic publishing and not necessarily aware of the differences between preprints and peer-reviewed publications, they might see findings 
from a preprint as solid scientific evidence rather than preliminary results that can still be altered or even disconfirmed following peer review. Scientists agree that one of the most significant challenges posed by preprints is the spread of unverified or incorrect information about essential topics, which might end up in the media as well (Chiarelli et al., 2019). Such risks were particularly troubling in the early stages of the COVID-19 pandemic when it was preprints rather than peer-reviewed studies that were driving the public discourse about the novel coronavirus (Majumder and Mandl, 2020).

In the case of the COVID-19 pandemic, the public discourse and the public opinion on the proposed measures have an important impact on their effectiveness. Until the production and distribution of vaccines, preemptive measures that reduce the number of contacts between infected and susceptible people, such as social distancing and wearing masks in public, have been the main strategy to curb the spread of the virus (Lewnard and Lo, 2020). Despite the robust evidence that, e.g., social distancing is helpful in this context (Koo et al., 2020), its effectiveness can be significantly undermined if the public does not comply with the measures (Maharaj and Kleczkowski, 2012). The same applies to the effectiveness of vaccines in curbing the pandemic: public distrust in vaccination will decrease compliance and vaccination rates, thus lowering the effectiveness of vaccines on the macro level.

\section{Politicization of Medical Information Online: COVID-19 and beyond}

In the last decade, certain health-related topics have become increasingly politicized and even weaponized online. One example of such a politically salient medical topic is vaccination with heated debates surrounding it on social media (Yuan et al., 2019). Previous studies, conducted before the COVID-19 pandemic, have found that vaccination-related discussions on social media are starkly polarized with echo chambering effects being present (Cossard et al., 2020; Schmidt et al., 2018). In addition, researchers found that Internet Research Agency - a Russian company that pursues online influence operations - was amplifying vaccine-related divisions on Twitter (Broniatowski et al., 2018; Walter et al., 2020) thus weaponizing the topic. Vaccination thus is a 
particularly politicized topic with (partisan) political cues having an influence on the people's opinion about this issue (Jones-Jang and Noland, 2020) ${ }^{1}$. That being said, the reach of bot-produced vaccine-skeptical information has been found to be limited (Dunn et al., 2020).

In the context of COVID-19 specifically, vaccine-related debates were also found to be highly politicized. $79 \%$ of those manifesting vaccine-skeptic opinions on Englishlanguage Twitter also expressed right-wing or conspiracy theory-driven views, and only $18 \%$ being politically neutral in their posting (Thelwall et al., 2021). Further, during the pandemic, citizens' willingness to adhere to preventative measures such as social distancing has been varying depending not only on the level of trust in science (Plohl and Musil, 2020) but also on political attitudes (Allcott et al., 2020; Funk and Tyson, n.d.; Painter and Qiu, 2020; Plohl and Musil, 2020). In the US, Republicans are less likely than Democrats to perceive social distancing as a helpful measure (Funk and Tyson, n.d.) and adhere to it (Allcott et al., 2020; Painter and Qiu, 2020).

The analysis of online conversations related to the COVID-19 pandemic has also demonstrated a high degree of (political) polarization in the way the issue is discussed in the US. For one, the online debates surrounding mask wearing are highly polarized (Lang et al., 2021). In addition, hashtags about preventative measures are twice as prevalent among Democrat and neutral Twitter users than among Republican users (Jiang et al., 2020).

\section{Research Questions}

Previous research shows that online discussions related to COVID-19 are highly politicized (Allcott et al., 2020; Painter and Qiu, 2020). At the same time, during COVID-19 the public has been increasingly engaging with academic preprints (Fraser et

\footnotetext{
${ }^{1}$ Politicization, however, is not universally manifested in relation to health-related topics, and some of the health-related debates tend to be unaffected by politics. For example, Twitter discussions about cancer have not been found to be politicized (Himelboim and Han, 2014).
} 
al., 2021), citing them more often than peer-reviewed studies in online debates related to COVID-19 (Majumder and Mandl, 2020). These two observations are the motivation behind the present study in which we examine the interconnections between the dissemination of medical preprints on social media and politicization of COVID-19related discussions. We assess the level of attention to medical preprints on Twitter during the COVID-19 pandemic and examine whether and how it is associated with the users' political partisanship. Specifically, we answer the following questions:

RQ1: Has the level of interest in medical preprints among Twitter users increased during the COVID-19 pandemic?

RQ2: Which medical preprints have attracted the most attention from Twitter users?

RQ3: Is there evidence of political partisanship in sharing patterns of COVID-19related preprints?

RQ4: Did the discourse about preprints among Twitter users change with the advent of COVID-19?

With this study, we aim to contribute to the existing body of scholarship related to the politicization of medical information in the online sphere. Below, we outline the methodology and report the results in connection to the Research questions outlined.

\section{Methods \\ Data collection}

The data includes all messages posted on Twitter that contained a medRxiv URL and were posted between June 2019 - the time when medRxiv was launched - and the end of June 2020. We thus include data from two roughly equal periods: one pre-COVID-19 (June 2019-January 2020), and one post the onset of COVID-19 (January-June 2020). We 
collected the data through Crimson Hexagon (currently known as Brandwatch) ${ }^{2}$ by requesting a full dataset of tweets that contain a link to medRxiv. This ensured a comprehensive list of tweet ids, which we then used to request complete tweet data through the Twitter API in May-June 2020. The resulting dataset contains 557405 tweets in total. The data includes all tweets that link to medRxiv (tweets, retweets, and quoted tweets) except for the tweets that were deleted or set as private between their creation and our retrieval date.

Of course, Twitter is just one of the multitude of online platforms and outlets where public discussions take place. Nonetheless, we believe that the platform is relevant in the context of the present study. Twitter along with other social media platforms is routinely used by the citizens to access news - in the US in $202047 \%$ of users have reported that they regularly get news from social media (Newman et al., 2020). Twitter posts can be used to gauge public sentiment on a given issue including COVID-19 (Valdez et al., 2020) as well as have real implications on the policy making processes either through the use of Twitter by journalists for public opinion mining (Beckers and Harder, 2016; Kim et al., 2015) or through direct communication between scientists, general public and health policy makers (Kapp et al., 2015).

\section{Analysis}

To address the research question concerning the increased interest in medical preprints among Twitter users, we calculate the number of tweets with links to medRxiv on Twitter by month. We then compare the numbers for the pre-pandemic and postpandemic periods. In addition, we control for the number of medRxiv preprints published during the same observation periods to assure that our observations stem from increased attention to preprints on Twitter and not simply from a surge in the number of medical preprints published.

\footnotetext{
${ }^{2}$ An analytics company that, among other, provided search for the full Twitter archive before Twitter Academic API was available.
} 
To check which preprints have attracted the most attention, we count the number of times each medRxiv preprint was shared on Twitter and then select the ten most frequently shared preprints.

To evaluate whether sharing patterns exhibit political partisanship, we first classify the users in the sample into four categories (Democrat, Republican, medical professional, scientist). Categorization of users into the four categories (Democrat, Republican, medical professional, scientist) was done by matching the terms in their user profile descriptions with four sets of keywords:

- Democrat-corresponding terms: DNC, Democrat, Democratic, Liberal, Biden

- Republican-corresponding terms: GOP, Trump, Republican, Conservative, MAGA

- Scientist-corresponding terms: PhD, University, Professor, Scientist, Researcher

- Medical professional-corresponding terms: Doctor, MD, Medicine, Medic, Medical

If a user had both Democrat- and Republican-corresponding terms in their profile, they were not classified as either.

Using the above-mentioned user classification, we compute the normalised share of interest of each user type for a specific time period. Intuitively, this metric compares the attention given to the respective preprint (or medRxiv in general) to the attention in a random sample for a specific type of user. We thus measure the level of observed partisanship not in absolute but in relation to a random sample of tweets that are not connected to medRxiv. This allows us to make sure that our observations - e.g., about disproportionate attention towards specific preprints from the aforementioned political groups - are robust, and not merely a by-product of the way we infer group membership. Further details on the procedure are listed in the Appendix.

Out of 259491 users who tweeted links to medRxiv in total, 1944 are classified as Democrats, 9174 - as Republicans, 8400 - as Medics, and 15400 - as Scientists. To compensate for the imbalance in the data with regard to the distribution of Democrats and Republicans, we normalized it by comparing it to the distribution drawn from a random sample of Twitter users. Notably, even after the normalization, our sample still appears to 
be heavily dominated by Republicans according to our classification. To assure that this observation reflects the underlying distribution correctly and is not erroneous due to our methodology, we have validated it using the methodology developed by Barberá (2015). This additional check has confirmed that the sample of medRxiv-linking users is heavily skewed towards conservatives as compared to a sample of random users (see the details in the Appendix). To further assure the robustness of our findings, we have checked whether they could have been affected by the presence of bots in our sample, and have concluded that the likelihood of this is low (we present the details on this check in the Appendix).

Then we compute the normalized share of interest towards medical preprints (based on the number of shared links to medRxiv preprints) from each of these categories of users. Finally, we calculate what we call the party share index (PSI) to establish whether sharing patterns of certain preprints have a strong Democratic or Republicanleaning. To calculate the PSI, we compute ratios of tweets by Democrats versus a random sample, repeat the computation for Republicans, and then calculate a PSI score as a logtransformed fraction of tweets by each of the two groups. For more details about the categorization of users and calculation of the normalized share of interest and PSI, see the Appendix.

To examine whether the interpretations of preprints by Twitter users have changed during the COVID-19 pandemic, we look at the way the percentage of tweets linking to medRxiv with certain keywords (preprint, review, study, paper, research, pdf) changed over time. Besides, we test whether different categories of users (i.e., Republicans, Democrats, Medics, Scientists) are more likely than other users to tweet one of these keywords when linking to medRxiv (see the Appendix for details on the calculations). Lastly, we look at word shift graphs to see whether the changes in the usage of keywords are explained by the shift in preprint audience. Word shift graphs are vertical bar charts that visualize which words and how much contribute to the differences between two sets of texts (Gallagher et al., 2021). In the present study, the differences are based on the comparison of the proportions of words in the tweets posted by Republicans and Democrats vs Medics and Scientists. 


\section{Results}

\section{Attention to medRxiv overtime}

To establish whether the interest among Twitter users towards medical preprints has grown during the pandemic, we look at the volume of tweets in our data set over time. Figure 1 shows the cumulative number of tweets linking to medRxiv for each timeperiod. Since the beginning of 2020, the number of tweets increased dramatically, peaking towards the end of April the same year. This surge overlapped time-wise with the increased concern about the COVID-19 pandemic. For the first few months, the number of shares of medRxiv preprints on Twitter was surging, similarly to the number of published preprints (Homolak et al., 2020), and in line with the observations about the public's increased engagement with preprints (Fraser et al., 2021). Around May, the number of tweets started decreasing, indicating a possible cool-down of interest in medRxiv among Twitter users. We have assured that this observation is due to the increased attention to medRxiv preprints on Twitter and not to the increase in the number of medRxiv preprints. For that, we have normalized the number of medRxiv-linking tweets by dividing it by the number of new medRxiv preprints ${ }^{3}$ published during the same observation period (Figure 2).

\footnotetext{
${ }^{3}$ medRxiv data was obtained using medrxivr R package (McGuinness \& Schmidt, 2020).
} 


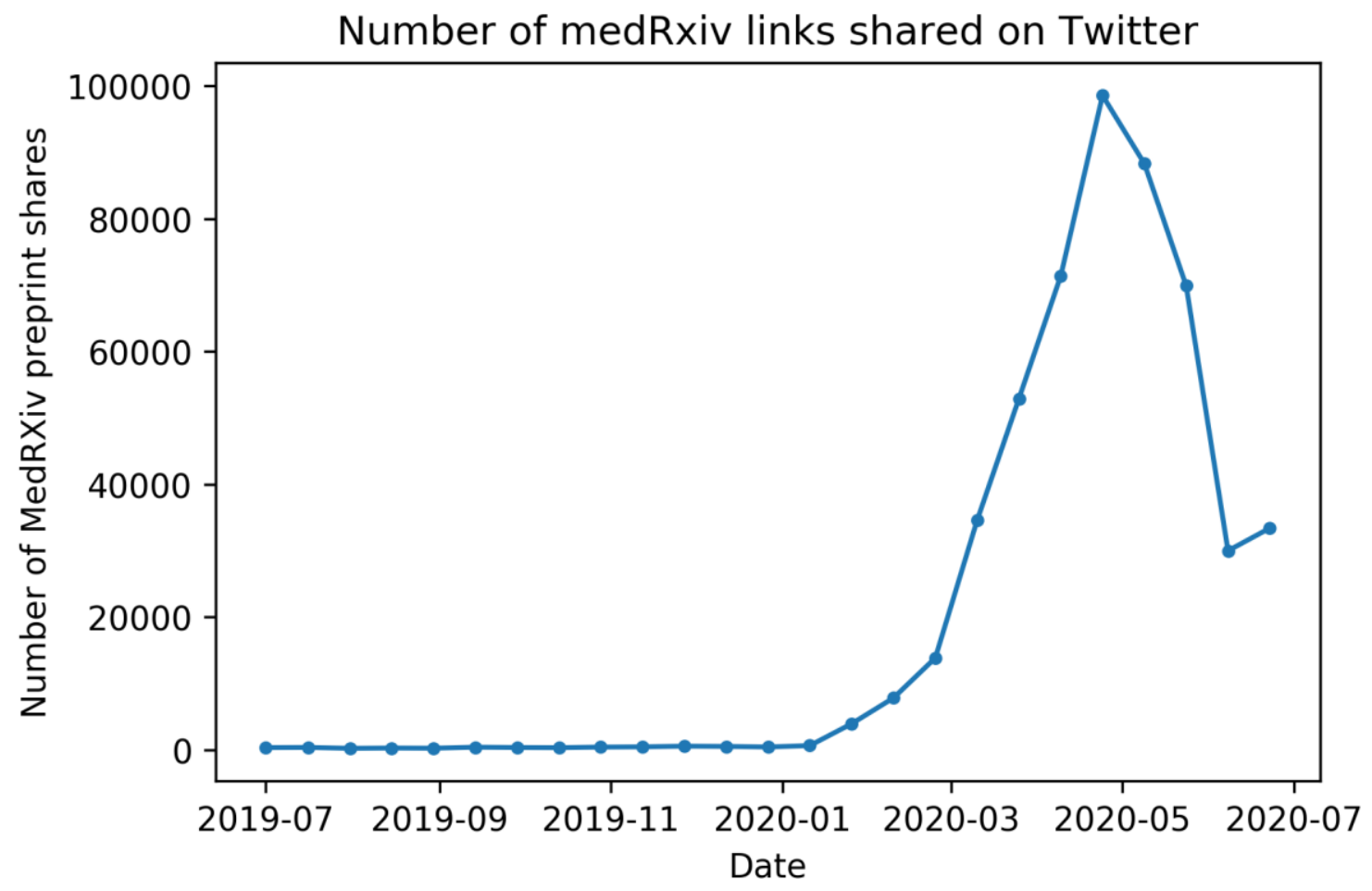

Figure 1. Number of medRxiv-linking tweets per time-period (one bin corresponds to approximately two weeks). 


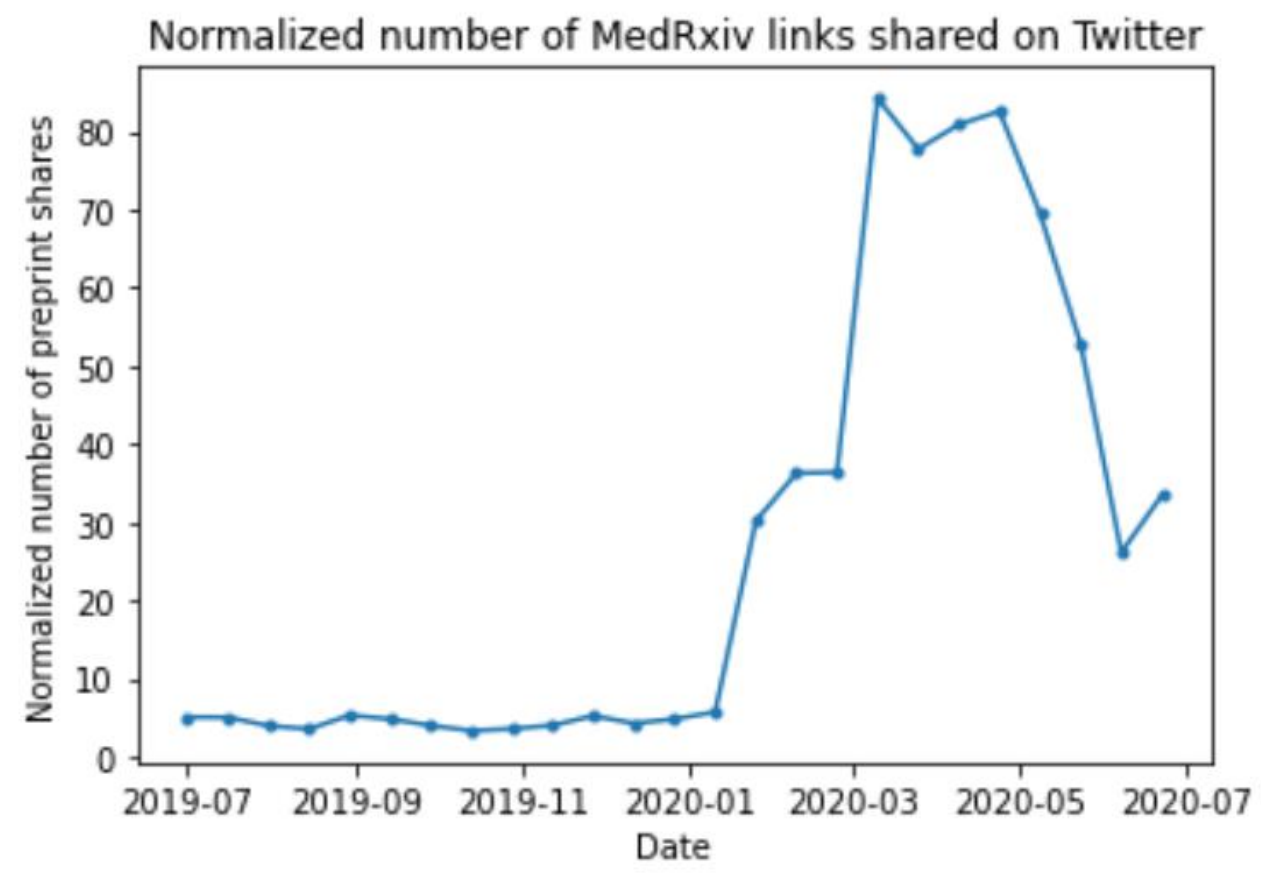

Figure 2. Normalized number of medRxiv-linking tweets per time-period.

Note. Normalization here means that the number of tweets with links to medRxiv divided by the number of new medRxiv preprints in each time period. One bin corresponds to approximately two weeks.

To make sure that the overlap in the increase in the number of tweets linking to medRxiv and the rising concern about the COVID-19 pandemic was not merely coincidental, we also look at the topics of the most cited preprints. Table 1 outlines the 10 most tweeted preprints. A qualitative examination of these preprints reveals they are all linked to COVID-19. In fact, all the 82 preprints that received at least 1000 shares are related to COVID-19; we refer the reader to the Appendix for the complete version of the table. Within the table, the preprints are ordered by the number of tweets mentioning them in our dataset.

Table 1. The ten most shared preprints on Twitter with the number of shares and the level of discourse partisanship. 
JQD: DM 2(2022)

Politicization of Medical Preprints

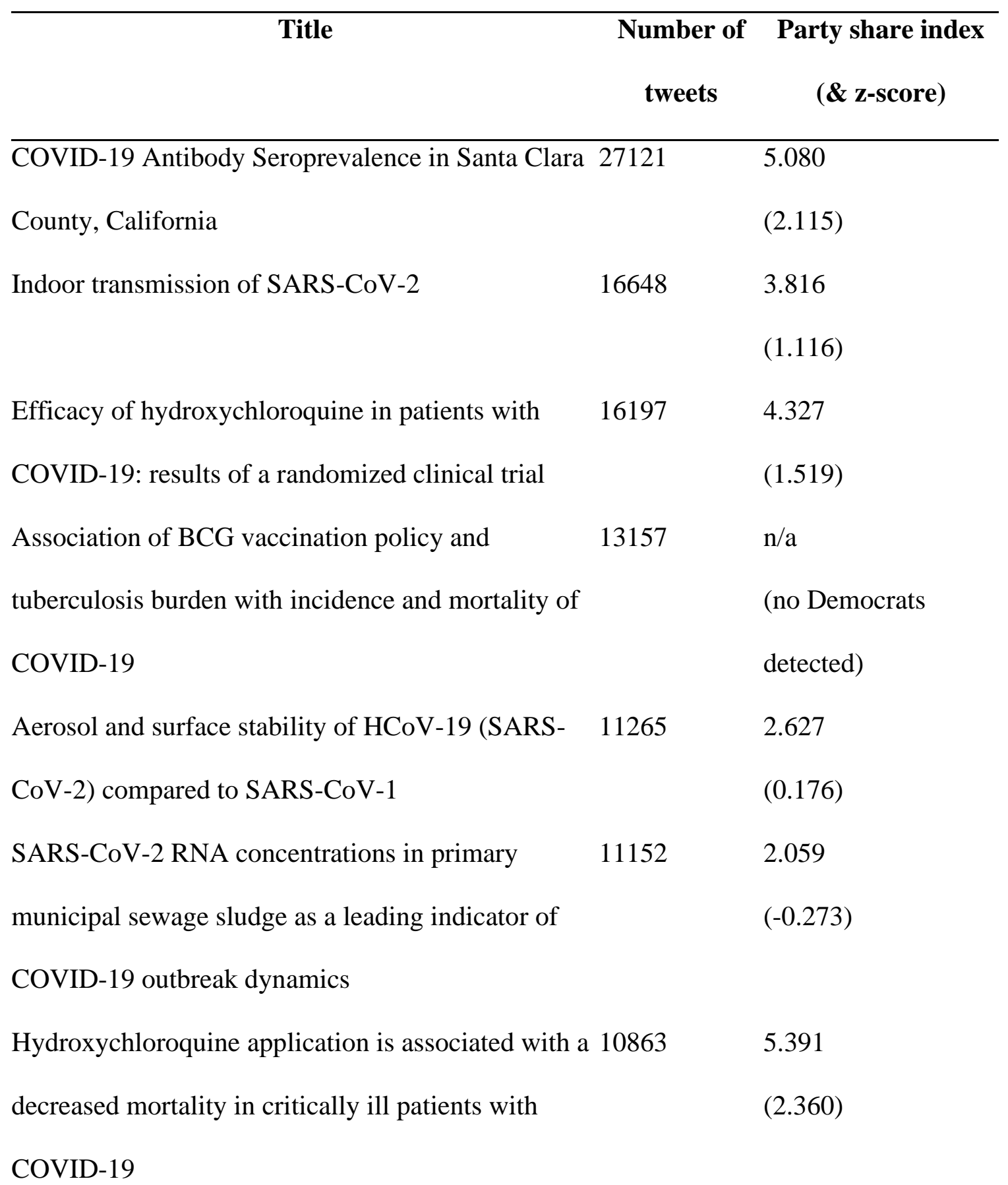


Hydroxychloroquine plus azithromycin: a potential 9386

interest in reducing in-hospital morbidity due to

COVID-19 pneumonia (HI-ZY-COVID)?

Outcomes of hydroxychloroquine usage in United $8623 \quad 3.598$

States veterans hospitalized

Hydroxychloroquine and azithromycin plus zinc vs $6763 \quad 6.131$

hydroxychloroquine and azithromycin alone:

outcomes in hospitalized COVID-19 patients

The analysis above indicates that the COVID-19 pandemic triggered an increase in the sharing volume of medical preprints. However, it is not only the raw number of links to medRxiv that changed. Figure 3 depicts the shift in the normalised share of interest of each of the four tracked types of users. Here we find the normalised share of interest per period. In particular, in each time interval we compare the fraction of users of a specific type that tweeted medRxiv links to the fraction of users of that type within a random sample of tweets. Consequently, if for example, Medics have a normalised share of interest of 0 in the second half of April, it means that the fraction of Medics tweeting about medRxiv in that time period is as high as in a random sample, i.e. they show the same amount of interest as within a random sample. Similarly, a negative value corresponds to a lower than random interest level, while a positive value corresponds to a higher than random interest level (more details on the calculations are available in the Appendix). 


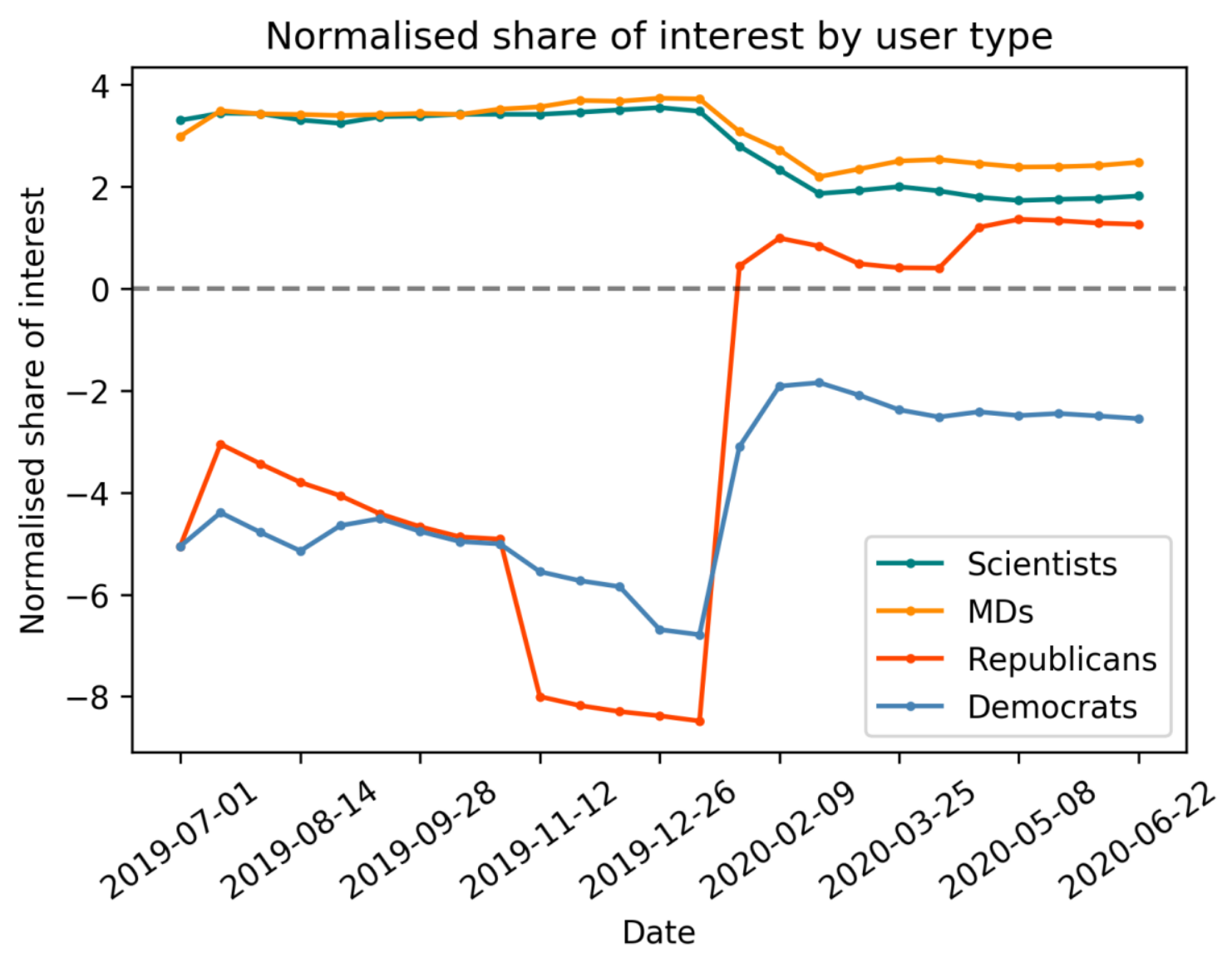

Figure 3. Normalised share of interest for medRxiv by user type.

With this interpretation in mind, Figure 3 reveals a shift in the interest of different types of users towards medRxiv with the increasing concern about the COVID-19 pandemic. If pre-pandemic - in the second half of 2019 - Medics and Scientists were the ones showing a higher than at random interest in medRxiv preprints, 2020 brought a dramatic increase in the strength of engagement from both Republicans and Democrats. Moreover, the fraction of Republicans tweeting medRxiv links increased to the level above what we find in a random sample. However, Democrats continued to show a lower level of sharing medRxiv papers when compared to the random sample. 
This brings an important change to the discourse around the preprints published on medRxiv. Before the COVID-19 pandemic, the sharing of these preprints on Twitter was mostly carried out by Medics and Scientists. However, more recently Republicans and Democrats have become an increasingly important part of the scene, indicating the politicization of the issue. In addition, political partisanship remains an influential factor differentiating between interest levels. A question that remains is whether this general trend concerning medRxiv preprint sharing on Twitter also persists in the case of individual preprints. To address this, in the next subsection, we look at individual preprints and the level of political partisanship in the context of their sharing patterns.

\section{Partisanship in the sharing patterns of individual medical preprints}

To identify the level of political partisanship in the sharing of preprints, we compare the ratio of Republicans to Democrats tweeting about a given preprint within our sample to the ratio within a random sample. This comparison is captured by a number, which we will refer to as the party share index (see the Methods Section for more details on how this metric is computed). A party share index close to zero signifies that the ratio of Republicans to Democrats is similar to the one within a random sample. Similarly, a negative value signals an unbalanced ratio, where the share of Democrat to Republican tweets is larger than within a random sample (Democrat-leaning sharing). In contrast, a positive value indicates a Republican-leaning sharing pattern. The further away a number is from zero, the more pronounced the political leaning is for a given preprint. Figure 4 shows the resulting distribution of preprints by party share index. For this analysis, we only include preprints that were shared by at least one Democrat and one Republican. For most preprints, Republicans are the ones with a stronger voice across shared preprints (mean $=2.4$, standard deviation $=1.27$ ). This result is in accordance with our analysis in the previous section, where we saw a significant difference in interest level between Republicans and Democrats. 


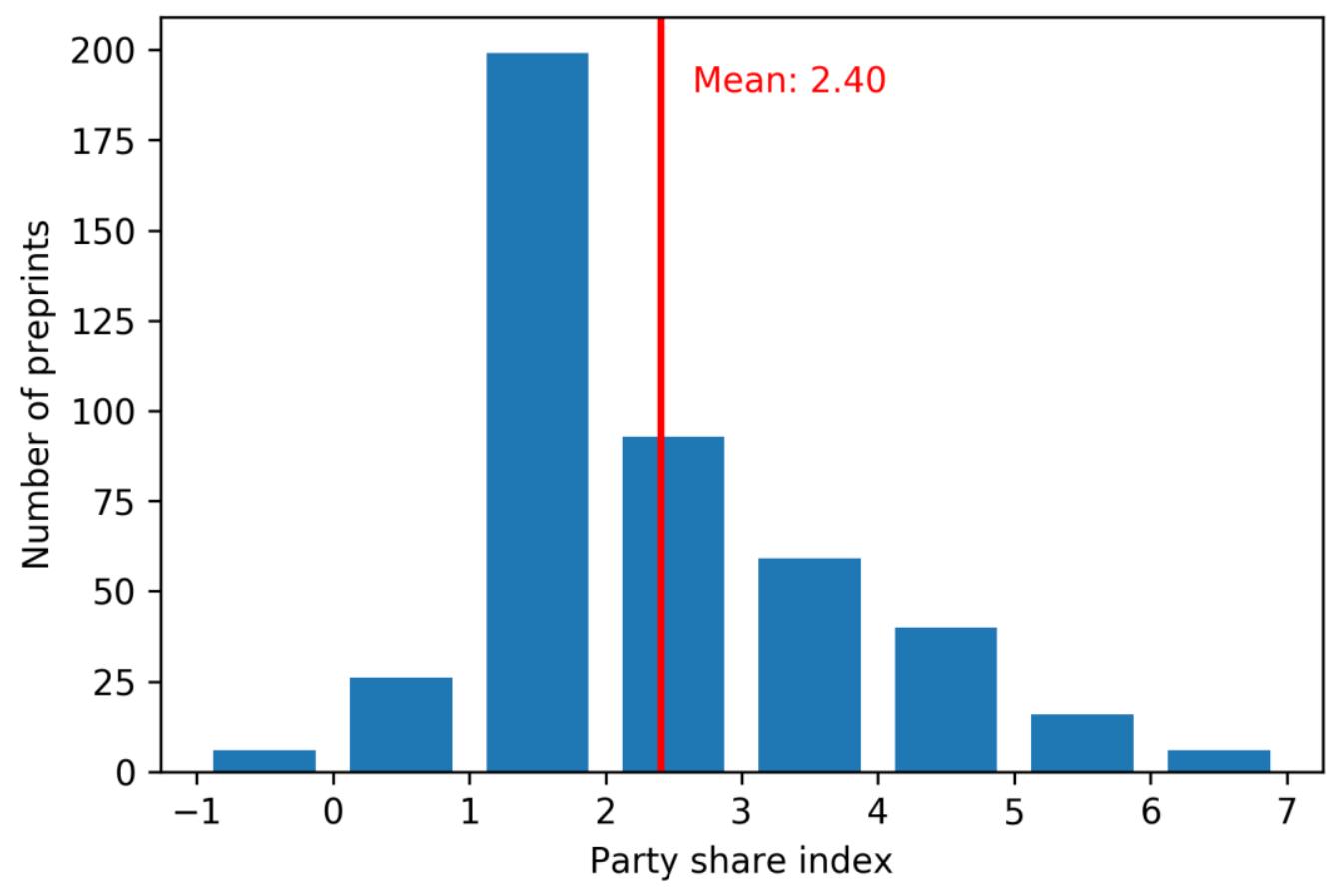

Figure 4. Party share index distribution for the preprints shared by at least one Democrat or one Republican. The vertical line corresponds to the mean.

To establish how strong a given preprint's sharing pattern was Democrat- or Republican-leaning, we use the z-score. This demonstrates how many standard deviations away from the mean a given preprint's party share index is. Table 1 shows the original party share indices as well as the accompanying z-scores for the top 10 most shared preprints. As shown in the table, most of the top 6 preprints in the list are close to the average level of partisanship, i.e. within two standard deviations from the mean. The fourth preprint is a special case, as it is shared by no Democrat in our dataset, so it has an undefined level of partisanship.

While among the top 10 most tweeted preprints there are many examples with Republican-leaning partisanship (i.e. having a z-score of at least 1), we see no case of 
Democrat-leaning partisanship in the top 10. The most shared Democrat-leaning preprint is titled "Effectiveness of isolation, testing, contact tracing and physical distancing on reducing transmission of SARS-CoV-2 in different settings" (1214 tweets, z-score = 2.399, party share index $=-0.631$ ).

So far, we have only looked at the changes over time in the party share interest at an aggregate level, without distinguishing by preprint. To see how sharing patterns of specific preprints by different user types change over time, we show temporal changes in the sharing of three example preprints with different party share indices. Figure 5 shows the time series for preprints 1, 2, and 6 where the order is taken from Table I. For the first preprint (Figure 5, on top), Republicans have the strongest interest since early April, i.e. the first time this preprint was shared. The numbers changed towards the end of June when Medics and Scientists had a lower than at random interest and the strength of the voice of Democrats raised considerably. A similar pattern is observed for the second preprint (Figure 5, in the middle). In the early stages of preprint dissemination on Twitter, Medics and Scientists engaged more than at random in the tweeting of this preprint. Later Republicans started to engage in the discussion more. A rise in the level of Democrat engagement only happened towards the end of the period. The last preprint we discuss (Figure 5, at the bottom), which has the lowest party share index, shows a different development. Through the period it was tweeted, it is mostly Medics and Scientists sharing this preprint, while the other two types of users demonstrated a lower than at random interest. Republicans were still more engaged than Democrats though, which is reflected in the positive value of the party share index for this preprint. 
JQD: DM 2(2022)
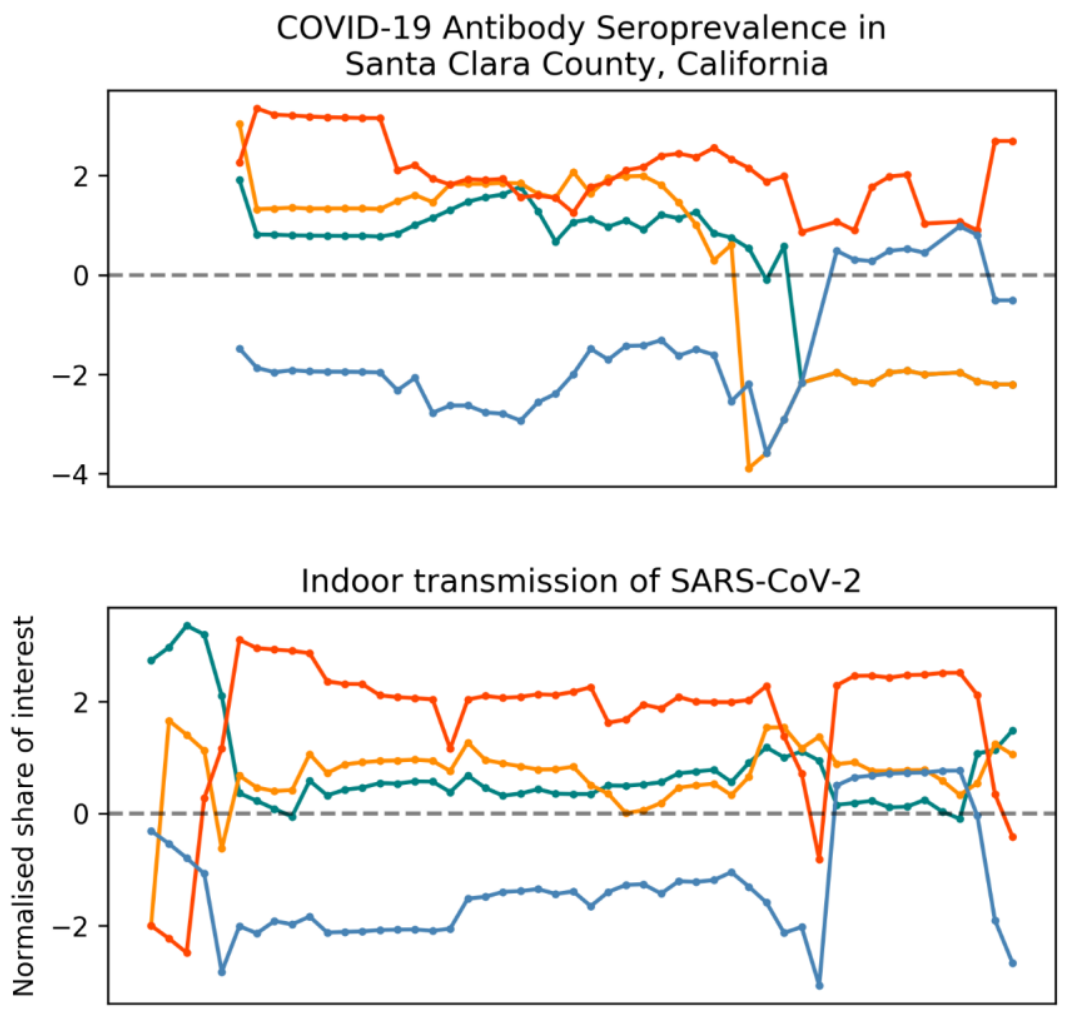

SARS-CoV-2 RNA concentrations in primary municipal sewage sludge as a leading indicator of COVID-19 outbreak dynamics

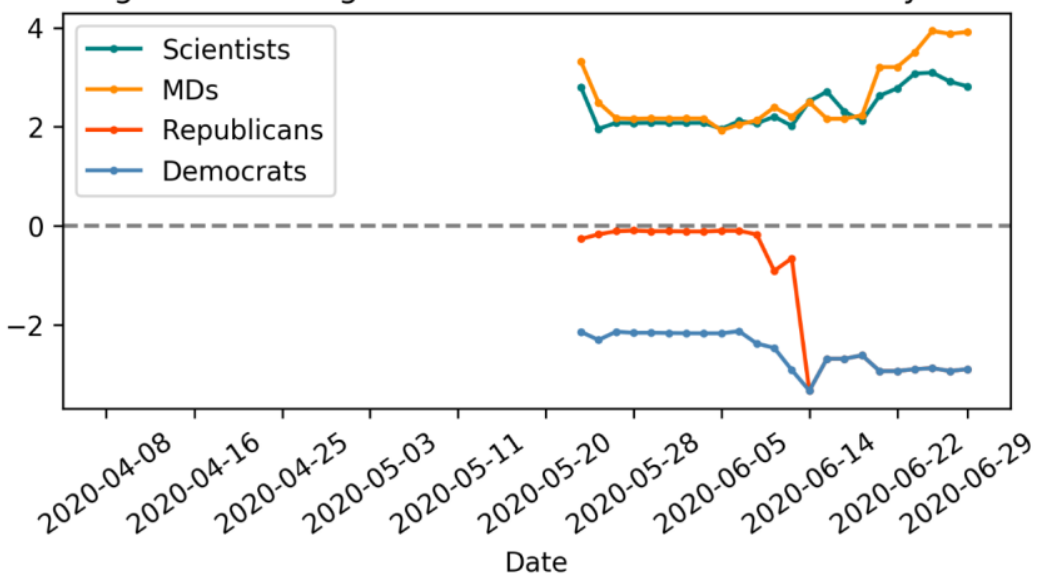

Figure 5. Normalised share of interest on Twitter by user type for three examples of preprints. 


\section{Discourse differences between user types}

Having seen which user types show an increased interest in tweeting different preprints, we turn our attention to how the discourse differs between them. First, we analyse the change in the terms used to refer to the preprint. Figure 6 shows the percentage of tweets containing each of the specified words in the respective time period. Prior to the COVID-19 pandemic, the term "preprint" was the one most frequently mentioned in tweets linking to medRxiv. After the onset of the pandemic, however, the share of tweets with "preprint" declined, and "study" became the most frequently mentioned term from our list. This shift happened simultaneously with the increase in public attention to medRxiv on Twitter, including the rise in the activity of politicallyengaged users, (Figures 1, 2, 3). This signifies the change in the overall discourse around medRxiv preprints on Twitter which was likely triggered by the increased interest in medRxiv from the general public. 
JQD: DM 2(2022)

Politicization of Medical Preprints
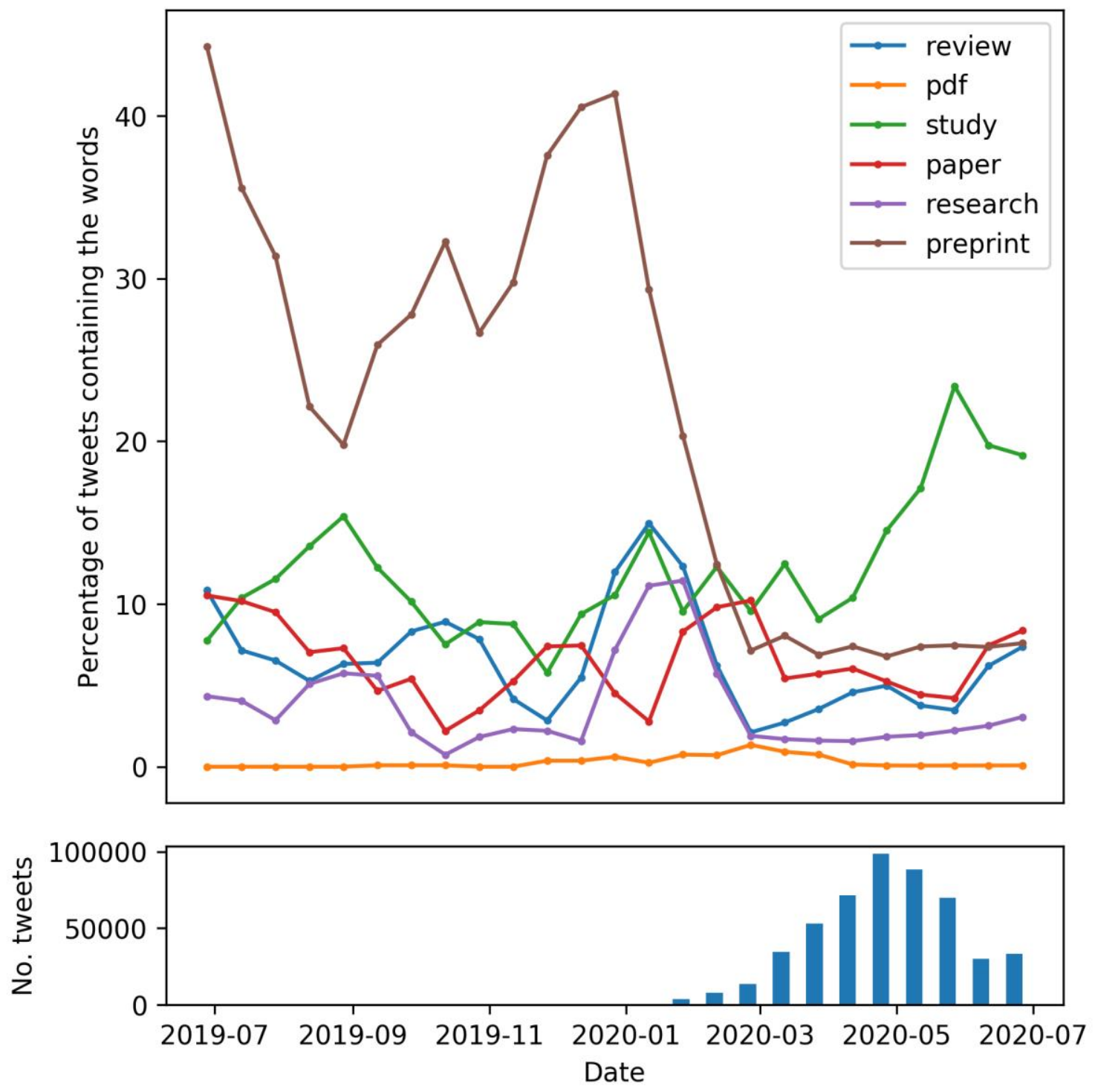

Figure 6. Percentages of tweets linking to medRxiv containing specific words

Before the pandemic when medRxiv links were tweeted mainly by the users identified as Medics or Scientists, users specified that the link they share is to a preprint, thus highlighting that the research in question is not peer-reviewed yet. After the onset of 
the pandemic, "study" became a more prevalent term than "preprint" in tweets linking to medRxiv. This suggests that after the onset of COVID-19 and the increase in the number of members of general public sharing links to medRxiv, non-peer-reviewed research papers started being referenced on Twitter more frequently as if they were properly validated scientific studies, without the mentions of the absence of a proper peer-review process.

To test whether this change in the word prevalence is driven by the increased interest from non-scientific and non-medical user types (general public), we identify the words that differ the most in the frequency with which they are used by different user types when discussing medRxiv preprints. As revealed by the word shift graph in Figure 7, Scientists and Medics differ from Republicans and Democrats in terms of the words they use when sharing medical preprints ${ }^{4}$. Two of the words the scientific and medical users differ the most from the political users, are "preprint" and "study". More precisely, Republicans and Democrats use "study" more often when sharing medRxiv links compared to Medics and Scientists who more frequently use "preprint" to refer to medRxiv content. This shows that indeed the change in word use when referring to medRxiv content is driven by the increased interest from political user types, which use different terminology when referring to preprints. Further, Democrats and Republicans were more likely than Medics and Scientists to refer to Donald Trump in their posts that included medRxiv links, indicating that their posts were more politicized.

\footnotetext{
${ }^{4} \mathrm{An}$ extended version of the graph in Figure 7 is available in the Appendix (see Figure A3).
} 


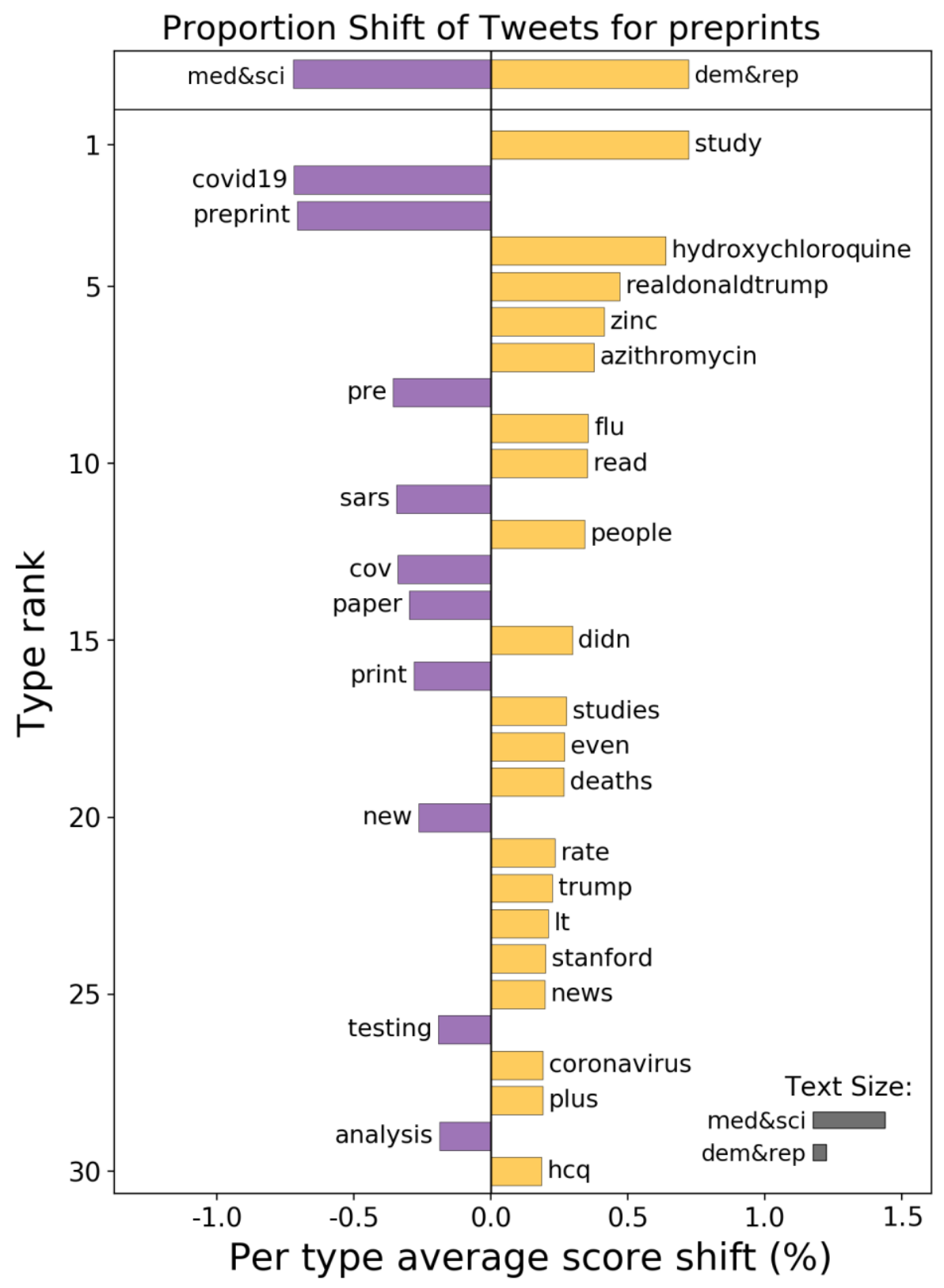

Figure 7. Word shift graph showing the words on which the Medics and Scientists differ the most from Democrats and Republicans in terms of usage frequency. 


\section{Limitations}

One limitation of the present study is that it relies on Twitter data, thus examining only one online platform which might not be representative of the whole online population. We find that for the purpose of the present study, Twitter data is suitable to capture general trends in online discourse concerning COVID-19. However, it is essential to examine other platforms such as Facebook or Telegram known for the amplification of conspiracy theories, including those related to the current pandemic (Bastani and Bahrami, 2020; Bruns et al., 2020; Urman and Katz, 2020).

Second, this study focuses on the US context. The developments occurring in the US are not universal and cannot be directly extrapolated to other countries. However, there is evidence that in other nations such as France and the Netherlands, the discourse surrounding COVID-19 is increasingly politicized. French and Dutch supporters of (far) right parties are more likely to believe in conspiracy theories about COVID-19 than their compatriots with different ideological orientations (Return of the paranoid style - Fake news is fooling more conservatives than liberals. Why? | International | The Economist, n.d.). And in Britain, in March 2020, supporters of the Conservative Party were way more likely to believe that COVID-19 was "just like the flu" than supporters of Labour (Return of the paranoid style - Fake news is fooling more conservatives than liberals. Why? | International | The Economist, n.d.). Right now, this leads to an ideological divide in the willingness of citizens to comply with preventative measures. Later on, this might also affect the citizens' willingness to vaccinate against COVID-19. There is mounting evidence that anti-vaccination discourse in connection to the current pandemic is getting traction online (Burki, 2020). We suggest that it is important to address the topic in future research on the online discourse concerning COVID-19, taking into account possible ideological divisions.

Finally, our way of identifying Democrat and Republican users is not fully precise as it relies on a rather crude proxy - mention of certain terms in users' Twitter bios. However, we suggest that together with the normalisation techniques used by us, this method and the results it provided still allow us to identify specific user groups with high 
enough precision for the purpose of the analysis performed in this study. This is confirmed by the robustness checks we performed relying on the method introduced by Barberá (2015), as noted in the Methodology and described in detail in the Appendix.

\section{Summary and Discussion}

Through the analysis of the medRxiv sharing patterns on Twitter, we have established that the COVID-19 pandemic has triggered an increase in interest towards medical preprints among Twitter users. Before the pandemic, medical preprints were predominantly shared by users we identify as medical professionals and scientists, and rarely shared by users we classify as political partisans. After the beginning of the pandemic, partisan users, especially Republicans, actively started sharing medical preprints (Figure 3). We thus observe politicization of preprint-sharing patterns on Twitter. As indicated by the party share index, the majority of most-tweeted preprints were shared by Republicans way more than by the Democrats. Qualitative analysis shows that preprints with the highest Republican-leaning as measured by the PSI are related to the use of hydroxychloroquine as a treatment for COVID-19. The results of the analysis of the keywords most frequently mentioned in tweets linking to medRxiv suggest that the general public and politicised groups tend to refer to medRxiv papers as regular scientific studies rather than preliminary results still pending peer review.

Medical preprints, when shared widely, can influence public opinion and thus have important implications for the spread of COVID-19 since the public needs to comply with preventive measures for them to be effective. During a pandemic such as COVID-19 scientific understanding of the nature and effects of the disease is rapidly evolving. In such a situation increased sharing of medical and scientific information among the general public before the information is properly vetted can lead to or exacerbate the infodemic (The Lancet Infectious Diseases, 2020). In the early stages of the pandemic, users turned to Twitter to seek out and promote advice on the disease (Cinelli et al., 2020). In general, though users turn to social media for actively seeking health-related information less frequently than to search engines, when they do, in over 
half of the cases it is to find information on disease treatments (De Choudhury et al., 2014). Further, social media users can encounter health-related information incidentally in their algorithmically curated feeds, even when they are not actively looking for it. In the context when scientific consensus about effective treatments is not established, sharing not properly validated findings on social media can be especially harmful. Users can come across conflicting claims or believe in the effectiveness of some treatments for which there is no evidence validated through peer-review. There is evidence that the users frequently do not fully read everything they encounter or share online (Dewey, n.d.; Gabielkov et al., 2016), in which cases they likely form their opinion about the content of the shared URL simply by the accompanying post or the links' headline. The fact that users do not always read what they share carefully makes the spread of unvalidated medical information potentially even more dangerous. Social media users rely on the headlines of the preprints they encounter or accompanying tweets not necessarily going into details on how a study was conducted or on potential side effects of any treatment proposed in the study. Further, when medical information is politicized, such unsubstantiated evidence from preprints can be amplified by political actors broadening the reach of such information and thus posing danger to public health. This was the case, for example, with the presumed effectiveness of hydroxychloroquine as a treatment for COVID-19 - a narrative that was pushed forward by Donald Trump with corresponding preprints being amplified by conservative public figures and journalists in our dataset. We have qualitatively examined most retweeted tweets with each of the most shared medRxiv preprints and found that users predominantly summarized the findings of said preprints or linked to them when discussing COVID-19-related measures. Tweets contesting preprint findings were absent at least among the most retweeted posts.

The increased interest in medRxiv from the general public and, especially, the politicization of preprint-sharing patterns, call for higher scrutiny when dealing with preprints, especially those concerning COVID-19. While we as a society expect scientific evidence to be the common ground that can be used to resolve disagreements, in practice we find that groups with different ideologies during the pandemic have been sharing different kinds of preprints - sometimes those with the opposite conclusions - regarding 
COVID-19. This highlights that scientific findings can be selectively used to advance various political interests and further exacerbate existing political divides instead of forming a common ground for different social and political groups. While this might be problematic even in the context of peer-reviewed findings that can be selectively picked up by people with strong ideological orientations to match their pre-existing attitudes, the issue is even more troubling in the case of preprints. While findings from preprints can be disproven during peer-review, the public is not always aware of the differences between peer-reviewed and non-peer-reviewed studies, and arguments put forward in preprints can be disseminated and used in political discussions even before they are properly verified - something scientists have long been concerned about in the context of preprints (Chiarelli et al., 2019).

If medical preprints are here to stay, their responsible handling is necessary to avoid possible harm in the future. This can be achieved only through active participation of all the parties involved. For scientists, it is important to consider whether the non-peerreviewed findings that they share could negatively affect public health if disconfirmed or altered at a later stage but shared widely before that. Same considerations should be taken into account by medRxiv and other preprint services before approving the publication of a given preprint. Since media have an extraordinary power to amplify scientific findings, journalists should be particularly careful when sharing non-peer-reviewed research and, at least, highlight the difference between a preprint and a peer-reviewed published study when doing so. Additionally, social media companies might consider implementing measures aimed at helping their users distinguish between fully validated and preliminary results of scientific research such as, for example, adding disclaimers to the posts containing links to medRxiv and preprint. This could be done by adding to preprintlinking tweets labels similar to those that Twitter started attaching to the government and state-affiliated media accounts in August 2020. Finally, as the discussions around COVID-19-related measures are highly politicised, political actors should use preprints responsibly in their rhetoric and avoid presenting non-peer-reviewed research as strong scientific evidence to the public, even if the findings of a preprint in question align with their political views. 


\section{Acknowledgments}

We thank the editors and the anonymous reviewers for their feedback that helped improve the original manuscript. We also thank Aleszu Bajak for the helpful comments on our ideas at the early stage of conceptualization of this manuscript.

\section{Funding}

The authors did not receive any dedicated funding for this research.

\section{References}

Allcott, H., Boxell, L., Conway, J., Gentzkow, M., Thaler, M. and Yang, D.Y. (2020), Polarization and Public Health: Partisan Differences in Social Distancing During the Coronavirus Pandemic, SSRN Scholarly Paper No. ID 3574415, Social Science Research Network, Rochester, NY, available at: https://papers.ssrn.com/abstract=3574415 (accessed 30 August 2020).

Barberá, P. (2015). Birds of the Same Feather Tweet Together: Bayesian Ideal Point Estimation Using Twitter Data. Political Analysis, 23(1), 76-91. https://doi.org/10.1093/pan/mpu011

Bastani, P. and Bahrami, M. (2020), “COVID-19 Related Misinformation on Social Media: A Qualitative Study from Iran.”, Journal of Medical Internet Research, available at:https://doi.org/10.2196/18932.

Beckers, K. and Harder, R.A. (2016), “"Twitter Just Exploded”, Digital Journalism, Routledge, Vol. 4 No. 7, pp. 910-920.

Berg, J.M., Bhalla, N., Bourne, P.E., Chalfie, M., Drubin, D.G., Fraser, J.S., Greider, C.W., et al. (2016), "Preprints for the life sciences", Science, American Association for the Advancement of Science, Vol. 352 No. 6288, pp. 899-901.

Broniatowski, D.A., Jamison, A.M., Qi, S., AlKulaib, L., Chen, T., Benton, A., Quinn, S.C., et al. (2018), "Weaponized Health Communication: Twitter Bots and 
JQD: DM 2(2022)

Politicization of Medical Preprints

Russian Trolls Amplify the Vaccine Debate", American Journal of Public Health, American Public Health Association, Vol. 108 No. 10, pp. 1378-1384.

Bruns, A., Harrington, S. and Hurcombe, E. (2020), “'Corona? 5G? or both?': the dynamics of COVID-19/5G conspiracy theories on Facebook", Media International Australia, SAGE Publications Ltd, Vol. 177 No. 1, pp. 12-29.

Burki, T. (2020), "The online anti-vaccine movement in the age of COVID-19", The Lancet Digital Health, Elsevier, Vol. 2 No. 10, pp. e504-e505.

Chiarelli, A., Johnson, R., Pinfield, S. and Richens, E. (2019), "Preprints and Scholarly Communication: An Exploratory Qualitative Study of Adoption, Practices, Drivers and Barriers", F1000Research, Vol. 8, available at:https://doi.org/10.12688/f1000research.19619.2.

Cinelli, M., Quattrociocchi, W., Galeazzi, A., Valensise, C.M., Brugnoli, E., Schmidt, A.L., Zola, P., et al. (2020), "The COVID-19 social media infodemic", Scientific Reports, Nature Publishing Group, Vol. 10 No. 1, p. 16598.

Cossard, A., Morales, G.D.F., Kalimeri, K., Mejova, Y., Paolotti, D. and Starnini, M. (2020), "Falling into the Echo Chamber: The Italian Vaccination Debate on Twitter", Proceedings of the International AAAI Conference on Web and Social Media, Vol. 14, pp. 130-140.

De Choudhury, M., Morris, M.R. and White, R.W. (2014), "Seeking and sharing health information online: comparing search engines and social media", Proceedings of the SIGCHI Conference on Human Factors in Computing Systems, Association for Computing Machinery, New York, NY, USA, pp. 1365-1376.

Dewey, C. (n.d.). "6 in 10 of you will share this link without reading it, a new, depressing study says", Washington Post, available at: https://www.washingtonpost.com/news/the-intersect/wp/2016/06/16/six-in-10-ofyou-will-share-this-link-without-reading-it-according-to-a-new-and-depressingstudy/ (accessed 27 January 2021). 
Dunn, A.G., Surian, D., Dalmazzo, J., Rezazadegan, D., Steffens, M., Dyda, A., Leask, J., et al. (2020), "Limited Role of Bots in Spreading Vaccine-Critical Information Among Active Twitter Users in the United States: 2017-2019”, American Journal of Public Health, American Public Health Association, Vol. 110 No. S3, pp. S319-S325.

Fraser, N., Brierley, L., Dey, G., Polka, J.K., Pálfy, M., Nanni, F. and Coates, J.A. (2021), "The evolving role of preprints in the dissemination of COVID-19 research and their impact on the science communication landscape", PLOS Biology, Public Library of Science, Vol. 19 No. 4, p. e3000959.

Funk, C. and Tyson, A. (n.d.). "Partisan Differences over the Pandemic Response Are Growing", Scientific American Blog Network, available at: https://blogs.scientificamerican.com/observations/partisan-differences-over-thepandemic-response-are-growing/ (accessed 30 August 2020).

Gabielkov, M., Ramachandran, A., Chaintreau, A. and Legout, A. (2016), "Social Clicks: What and Who Gets Read on Twitter?", presented at the ACM SIGMETRICS / IFIP Performance 2016, available at: https://hal.inria.fr/hal-01281190 (accessed 27 January 2021).

Gallagher, R.J., Frank, M.R., Mitchell, L., Schwartz, A.J., Reagan, A.J., Danforth, C.M. and Dodds, P.S. (2021), "Generalized word shift graphs: a method for visualizing and explaining pairwise comparisons between texts", EPJ Data Science, Vol. 10 No. 1, p. 4.

Himelboim, I. and Han, J.Y. (2014), "Cancer Talk on Twitter: Community Structure and Information Sources in Breast and Prostate Cancer Social Networks", Journal of Health Communication, Taylor \& Francis, Vol. 19 No. 2, pp. 210-225.

Homolak, J., Kodvanj, I. and Virag, D. (2020), "Preliminary analysis of COVID-19 academic information patterns: a call for open science in the times of closed borders", Scientometrics, Vol. 124 No. 3, pp. 2687-2701.

Jiang, J., Chen, E., Yan, S., Lerman, K. and Ferrara, E. (2020), “Political polarization 
JQD: DM 2(2022)

Politicization of Medical Preprints

drives online conversations about COVID-19 in the United States", Human Behavior and Emerging Technologies, Vol. 2 No. 3, pp. 200-211.

Jones-Jang, S.M. and Noland, C. (2020), "The Politicization of Health and Science: Role of Political Cues in Shaping the Beliefs of the Vaccine-Autism Link", Health Communication, Routledge, Vol. 0 No. 0, pp. 1-9.

Kapp, J.M., Hensel, B. and Schnoring, K.T. (2015), "Is Twitter a forum for disseminating research to health policy makers?", Annals of Epidemiology, Vol. 25 No. 12, pp. $883-887$.

Kim, Y., Kim, Y., Lee, J.S., Oh, J. and Lee, N.Y. (2015), “Tweeting the public: journalists' Twitter use, attitudes toward the public's tweets, and the relationship with the public", Information, Communication \& Society, Routledge, Vol. 18 No. 4, pp. 443-458.

Koo, J.R., Cook, A.R., Park, M., Sun, Y., Sun, H., Lim, J.T., Tam, C., et al. (2020), "Interventions to mitigate early spread of SARS-CoV-2 in Singapore: a modelling study", The Lancet Infectious Diseases, Elsevier, Vol. 20 No. 6, pp. 678-688.

Kupferschmidt, K. (2020), "Preprints bring 'firehose' of outbreak data", Science, American Association for the Advancement of Science, Vol. 367 No. 6481, pp. 963-964.

Lang, J., Erickson, W.W. and Jing-Schmidt, Z. (2021), “\#MaskOn! \#MaskOff! Digital polarization of mask-wearing in the United States during COVID-19", PLOS ONE, Public Library of Science, Vol. 16 No. 4, p. e0250817.

Lewnard, J.A. and Lo, N.C. (2020), "Scientific and ethical basis for social-distancing interventions against COVID-19", The Lancet. Infectious Diseases, Vol. 20 No. 6, pp. 631-633.

van der Linden, S. van der, Roozenbeek, J., Maertens, R., Basol, M., Kácha, O., Rathje, S. and Traberg, C.S. (2021), "How Can Psychological Science Help Counter the Spread of Fake News?", The Spanish Journal of Psychology, Cambridge 
University Press, Vol. 24, available at:https://doi.org/10.1017/SJP.2021.23.

Maharaj, S. and Kleczkowski, A. (2012), "Controlling epidemic spread by social distancing: Do it well or not at all”, BMC Public Health, Vol. 12 No. 1, p. 679.

Majumder, M.S. and Mandl, K.D. (2020), "Early in the epidemic: impact of preprints on global discourse about COVID-19 transmissibility", The Lancet Global Health, Elsevier, Vol. 8 No. 5, pp. e627-e630.

McGuinness, L. A., \& Schmidt, L. (2020). medrxivr: Accessing and searching medRxiv and bioRxiv preprint data in R. Journal of Open Source Software, 5(54), 2651. https://doi.org/10.21105/joss.02651

Newman, N., Fletcher, R., Schulz, A., Andi, S. and Nielsen, R.K. (2020), "Reuters Institute Digital News Report", Reuters Institute Digital News Report, available at: https://www.digitalnewsreport.org/ (accessed 18 January 2021).

Painter, M. and Qiu, T. (2020), Political Beliefs Affect Compliance with COVID-19 Social Distancing Orders, SSRN Scholarly Paper No. ID 3569098, Social Science Research Network, Rochester, NY, available at:https://doi.org/10.2139/ssrn.3569098.

Plohl, N. and Musil, B. (2020), "Modeling compliance with COVID-19 prevention guidelines: the critical role of trust in science", Psychology, Health \& Medicine, Taylor \& Francis, Vol. 0 No. 0, pp. 1-12.

"Return of the paranoid style - Fake news is fooling more conservatives than liberals. Why? | International | The Economist". (n.d.)., available at: https://www.economist.com/international/2020/06/03/fake-news-is-fooling-moreconservatives-than-liberals-why (accessed 16 October 2020).

Schmidt, A.L., Zollo, F., Scala, A., Betsch, C. and Quattrociocchi, W. (2018), "Polarization of the vaccination debate on Facebook", Vaccine, Vol. 36 No. 25, pp. 3606-3612. 
JQD: DM 2(2022)

The Lancet Infectious Diseases, E. (2020), "The COVID-19 infodemic", The Lancet Infectious Diseases, Elsevier, Vol. 20 No. 8, p. 875.

Thelwall, M., Kousha, K. and Thelwall, S. (2021), "Covid-19 vaccine hesitancy on English-language Twitter", Profesional de la información (EPI), Vol. 30 No. 2, available at:https://doi.org/10.3145/epi.2021.mar.12.

Urman, A. and Katz, S. (2020), "What they do in the shadows: examining the far-right networks on Telegram", Information, Communication \& Society, Routledge, Vol. 0 No. 0, pp. 1-20.

Valdez, D., Thij, M. ten, Bathina, K., Rutter, L.A. and Bollen, J. (2020), "Social Media Insights Into US Mental Health During the COVID-19 Pandemic: Longitudinal Analysis of Twitter Data", Journal of Medical Internet Research, Vol. 22 No. 12, p. e21418.

Walter, D., Ophir, Y. and Jamieson, K.H. (2020), "Russian Twitter Accounts and the Partisan Polarization of Vaccine Discourse, 2015-2017”, American Journal of Public Health, American Public Health Association, Vol. 110 No. 5, pp. 718-724.

Yuan, X., Schuchard, R.J. and Crooks, A.T. (2019), "Examining Emergent Communities and Social Bots Within the Polarized Online Vaccination Debate in Twitter", Social Media + Society, SAGE Publications Ltd, Vol. 5 No. 3, p. 2056305119865465. 


\section{Appendix}

\section{Further details on methodology}

Computing the normalized share of interest. The first step in computing this number is finding the share of interest of each user type (as described in the Methodology in the manuscript) in a random sample. To do so, we used a collection $(n=849,736)$ of random user bios - short descriptions of themselves that users can post on their Twitter accounts. The bios were collected the following way - we collected random tweets $(n=10,000)$ posted in the US per every day between January and the end of June 2020, then extracted user ids of the users who posted these tweets, removed duplicate ids, and extracted user bios. Then, based on the bios, we categorized the users in the same way as with the users in our sample. The share of interest of category $c$ in the random sample was then obtained by dividing the number of tweets of the people within the category by the total number of tweets:

$$
\begin{aligned}
& i_{c}^{r}=(\# \text { tweets in the random sample from users within category } c) / \\
& \text { (\# tweets in the random sample) } \\
& \qquad i_{c}^{r}=\frac{n_{c}^{r}}{n^{r}}
\end{aligned}
$$

Similar reasoning gives us the share of interest for a given topic (i.e. for a specific preprint or for medRxiv in general) within a given period of time. Therefore, similarly, the share of interest for the given topic, $t$, within category $c$ and within period $p$ is:

$$
\begin{aligned}
& i_{c(p)}^{t}=(\text { \# tweets on topic } t \text { within period p from users within category } c) \text { / } \\
& \text { (\# tweets on topic } t \text { within period } p) \\
& \qquad i_{c(p)}^{t}=\frac{n_{c(p)}^{t}}{n^{t(p)}}
\end{aligned}
$$

To obtain the normalised share of interest we divide the two above-introduced numbers and take logarithms. Hence, the normalised share of interest for topic $t$ within category $c$ in time period $p$ is:

$$
I_{c(p)}^{t}=\log _{2}\left(\frac{i_{c(p)}^{t}}{i_{c}^{r}}\right)
$$


This means that a value smaller than 0 corresponds to a lower than at random interest level while a larger value corresponds to a higher than at random interest level.

Arithmetically, there are two more details to consider. First, short time periods might result in unsmooth plots that reflect the granular behavior more than the overall trend in interest. Consequently, in computing $i_{c(p)}^{t}$ instead of only taking the numbers within time period $p$ we take the average over a few time periods around $p$. More precisely, for each plot we break the overall time frame of the tweets on topic $t$ into 50 equally-sized non-overlapping time-intervals, obtaining thus $p_{0}, p_{1}, \ldots, p_{24}$, and instead of only taking the row numbers of tweets on topic $t$ in period $p_{k}$, i.e. $n_{c\left(p_{k}\right)}^{t}$, we take the average that number and the (at most) 7 other ones immediately preceding. Therefore, the share of people in category $c$ tweeting about topic $t$ in time period $p_{k}$ is in fact:

$$
i_{c\left(p_{k}\right)}^{t}=\frac{n_{c}^{t}\left(p_{(0, k-7)}\right)+\ldots+n_{c}^{t}\left(p_{k}\right)}{n_{c}\left(p_{(0, k-7)}\right)+\ldots+n_{c}\left(p_{k}\right)}
$$

Second, the division and logarithm taking should be well-defined. In order to do

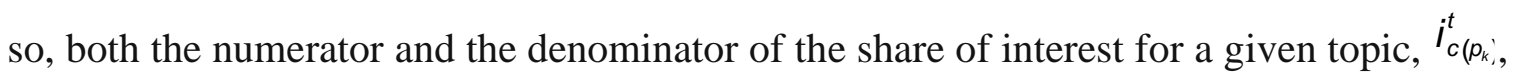
should be non-zero. To ensure this, we add an offset to the raw numbers of tweets. More precisely, we add 10 more fictive users, out of which the proportion in the random sample are of each category, i.e.:

$$
i_{c\left(p_{k}\right)}^{t}=\frac{n_{c}^{t}\left(p_{(0, k-7)}\right)+\ldots+n_{c}^{t}\left(p_{k}\right)+10 * i_{c}^{r}}{n^{t}\left(p_{(0, k-7)}\right)+\ldots+n^{t\left(p_{k}\right)}+10}
$$

Let us consider an example to show the workings of the offset. For simplicity, we only consider the political side. Therefore, there are three disjoint categories of agents: Republicans, Democrats, or None of the two. Let us further assume there are 10 people all identified as None. With the formula above, we get the following share of interests for each category: 


$$
i_{d e m}^{t}=i_{r e p}^{t}=\frac{0+\frac{10 * 1}{4}}{10+10}=\frac{2.5}{20}
$$

At this point, please note that the sum of shares is 1 , and thus preserved by adding the offsets. To obtain the normalised share of interest by user type, we divide by the respective shares within a random sample and taking logarithms, thus obtaining:

$$
I_{d e m}^{t}=I_{r e p}^{t}=\log _{2}\left(\frac{\left(\frac{2.5}{20}\right)}{\frac{1}{4}}\right)=-1
$$

Two important properties should be noted here. First, we can always take the logarithms after adding the offsets. Second, the meaning of 0 , negative, and positive values are preserved, as adding the offset is never changing the sign of the normalised share of interest.

Party share index. The discourse around a topic is composed of the voices of the people contributing to the discussion. Therefore, an imbalance in the political leaning of the users tweeting about a topic could indicate partisanship in the overall discussion of the respective topic. We capture this by party share index, a metric that compares the difference in the share of Republicans to Democrats in the respective discourse to the share within a random sample. More precisely, this level is defined as:

More precisely, this level is defined as:

$$
d^{t}=\log _{2}\left(\frac{n_{r e p}^{t} / n_{d e m}^{t}}{n_{r e p}^{t} / n_{d e m}^{\prime}}\right)
$$

where, as before, $n_{c}^{t}$ denotes the number of users in category $c$ tweeting about topic $t$, and $n_{c}^{r}$ denotes the number of users in category $c$ tweeting within the random sample. Similarly to the normalised share of interest, this formula is only well defined as long as there is at least one Republican and at least one Democrat tweeting about topic $t$. In this case, instead of adding an offset, we use n/a for the level of partisanship in 
preprints that don't have at least one Democrat and one Republican. Out of the 7,508 preprints shared in our dataset, 445 have specified levels of partisanship.

Validating the observed distribution of Republicans vs Democrats. In November 2021 - several months after the initial analysis within the paper was conducted - we implemented an additional check to validate that our sample is indeed dominated by Republicans, and that the observed distribution does not arise from errors or biases in our methodology for the detection of Republicans and Democrats. This was done using Barbera's (2015) method for estimating ideology of Twitter users. The method relies on the lists of accounts each user follows. We used this method as a check on subsamples of users and did not implement it for the full sample due to the Twitter Academic API's rate limits: retrieving the lists of followed accounts for all users in our medRxiv sample $(n=259491)$ and random sample $(n=849,736)$ would have taken several months of the data collection.

We included one random sample of non-medRxiv-linking users as a baseline 10000 accounts randomly selected from our sample of 849,736 accounts. Out of those we retrieved the lists of accounts followed by them for 7550 users (the remaining 2450 users had their accounts deleted or set to private by the time of the data collection in November 2021). We compared the ideology score distribution in this random sample with that among 10000 randomly selected medRxiv-linking users (accounts followed retrieved for 8708, the remaining 1292 accounts were deleted or set to private by November 2021). Further, we included a sample of all users who shared one of the preprints listed in Table A1; for these we had accounts followed collected in July 2020, with the data available for 17111 such users in total at that point. We suggest that this sample's ideology scores are more reflective of the real distribution during the observation period since the data was collected right after it. However, these scores are not entirely comparable to the random sample of non-medRxiv-linking users for who we only collected the data in November 2021: between July 2020 and November 2021 not only users' following patterns could have changed, a lot of accounts of conservative users - most notably that of Donald Trump - were suspended or deleted, inevitably shifting all the observations to the left. 
This was the reason why we included a second sample of medRxiv-linking users with the data collected in November 2021. We suggest this one is the most comparable to the random baseline, even if both samples are expected to be skewed to the left compared with that for which the data was collected in July 2020 . Hence, there were three samples of users analyzed using Barbera's (2015) method:

- 7550 randomly selected Twitter users (data collected in November 2021)

- 8708 randomly selected medRxiv-linking users (data collected in November 2021)

- 17711 users sharing one of the medRxiv preprints tweeted $>1000$ times between June 2019 and June 2020 (data collected in July 2020)

After the data was collected we estimated the ideologies of users in each of the samples using tweetscores package that implements Barbera's (2015) methodology specifically, estimateIdeology2 function was used. As shown in the Figure A1 below, both medRxiv-tweeting samples of users are heavily towards the $>0$ scores - those corresponding to conservatives, while the random sample is more skewed towards the $<0$ scores - those corresponding to liberals. As expected, the sample with the data collected in July 2020 is skewed to the right more prominently. However, given that the November 2021 sample of medRxiv-linking users is also skewed to the right compared to the random sample of non-medRxiv-linking users, we argue this additional check confirms that medRxiv-tweeting sample is skewed towards conservatives (or Republicans in our classification), in line with our initial analysis. 


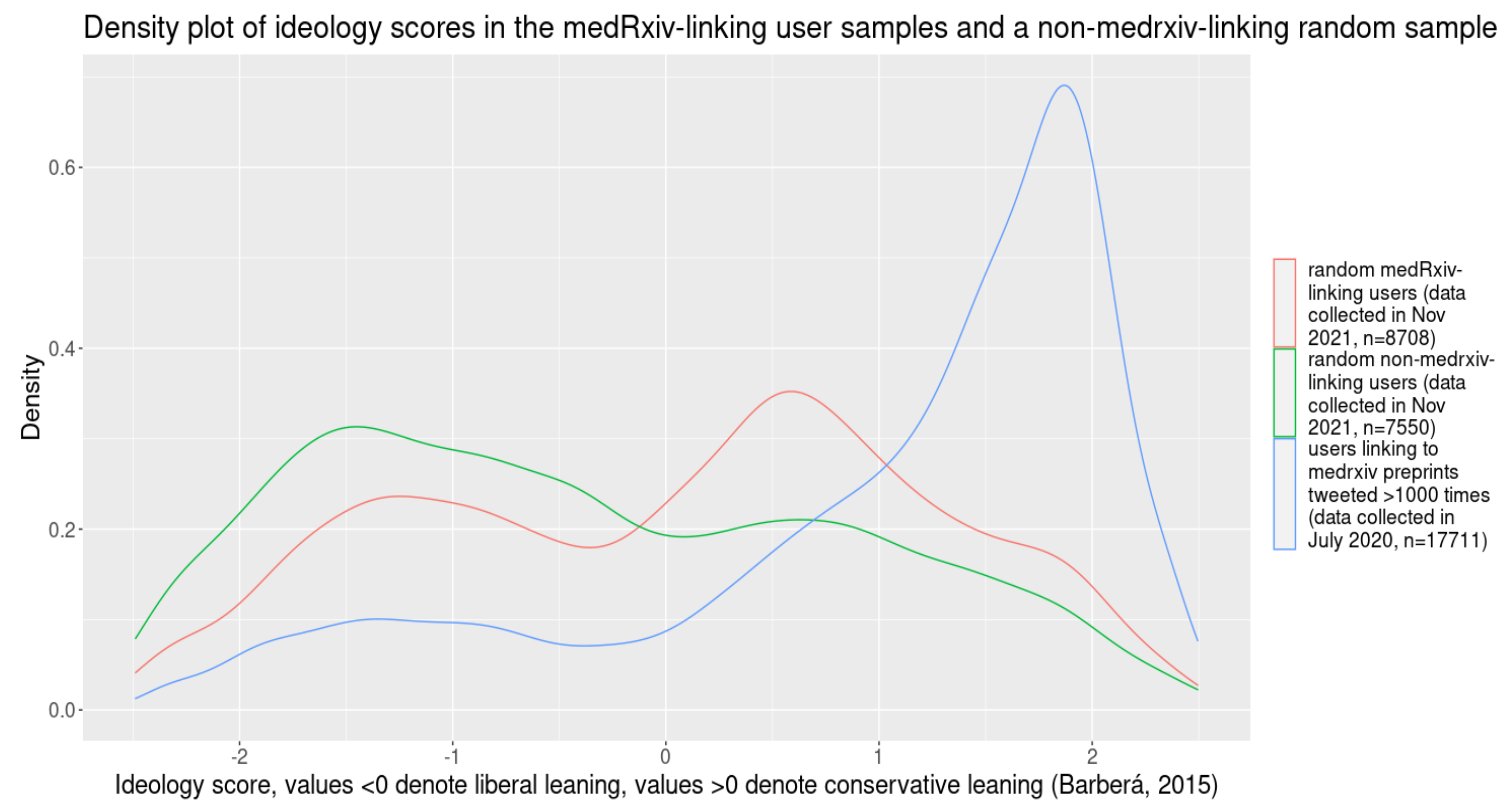

Figure A1. Density plot of ideology scores in the medRxiv-linking user sample and random sample based on Barbera's (2015) ideology detection methodology.

Checking for the presence of bots. Another robustness check implemented in November 2021 after the initial analysis was conducted was making sure that our observations correspond to real users and are not distorted by bots. For that, we have checked the sample using Botometer (https://botometer.osome.iu.edu). While we are aware of the critiques of the tool (e.g., Rauchfleisch \& Kaiser, 20205), we deemed it suitable for our purposes as we employed it only for a general check of the sample, bot presence or absence is not the focus of our paper. We ran the Botometer on the sample of medRxiv-linking users $(n=203277$ - the number of users from our original sample whose accounts were not set to private or deleted at the time of the data collection in November 2021). As shown in Figure A2, the share of users for who the probability of being a bot is estimated as high is marginal in our sample (3\% of users with Botometer scores $>0.5$ and $0.2 \%$ with Botometer scores $>0.8$ ). Based on this along with the fact that Botometer is

\footnotetext{
${ }^{5}$ Rauchfleisch, A., \& Kaiser, J. (2020). The False Positive Problem of Automatic Bot Detection in Social Science Research (SSRN Scholarly Paper ID 3565233). Social Science Research Network. https://doi.org/10.2139/ssrn.3565233
} 
more likely to return false positives than false negatives (Rauchfleisch \& Kaiser, 2020), we conclude that the likelihood of our observations being distorted by bots is low.

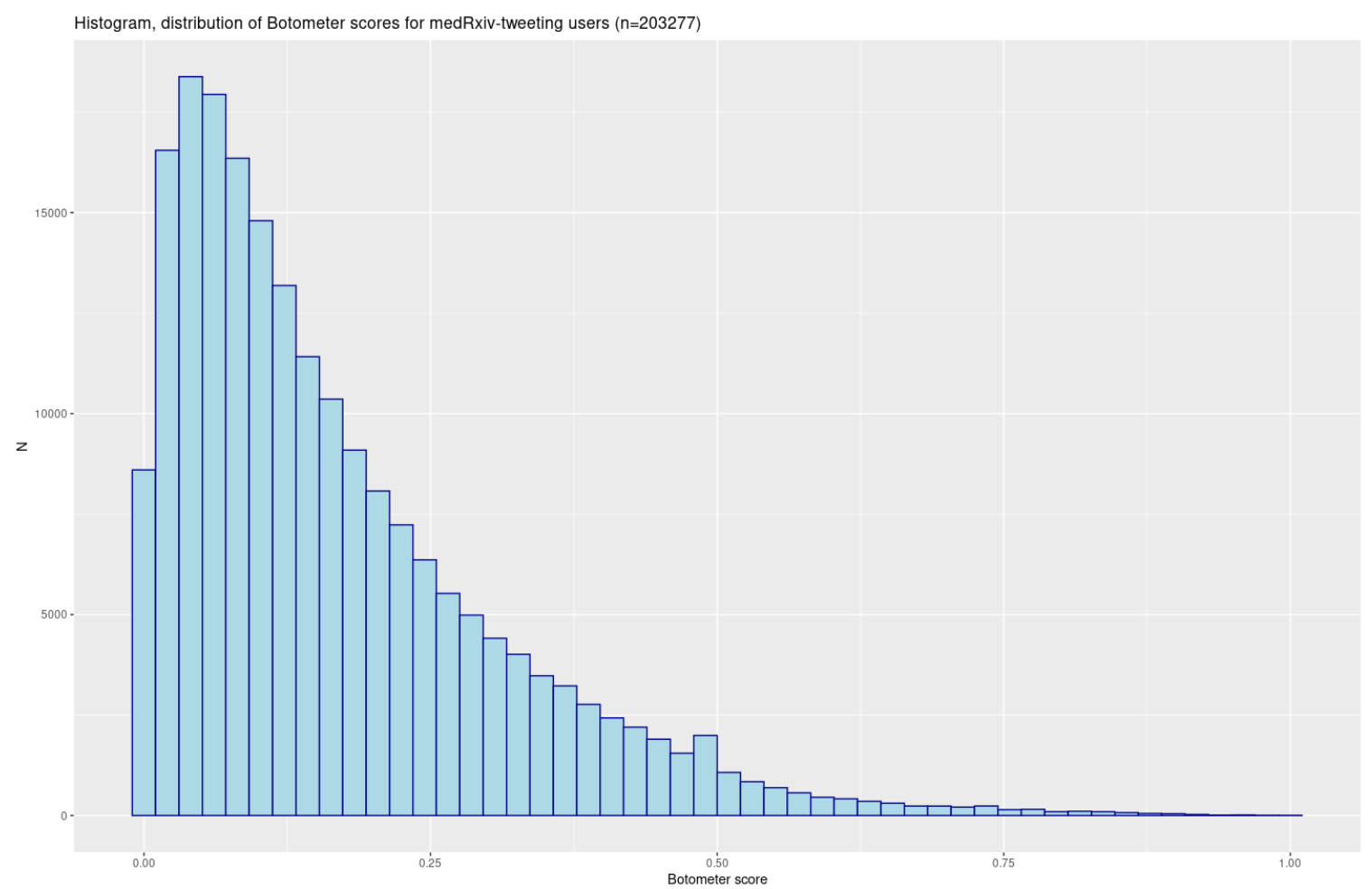

Figure A2. Distribution of Botometer scores.

Table A1. Full list of preprints tweeted at least 1000 times

\begin{tabular}{ll}
\hline \multicolumn{1}{c}{ Preprint title } & No. Shares \\
\hline COVID-19 Antibody Seroprevalence in Santa Clara County, California & 27121 \\
Indoor transmission of SARS-CoV-2 & 16648 \\
$\begin{array}{l}\text { Efficacy of hydroxychloroquine in patients with COVID-19: results of a } \\
\text { randomized clinical trial }\end{array}$ & 16197 \\
$\begin{array}{l}\text { Association of BCG vaccination policy and tuberculosis burden with } \\
\text { incidence and mortality of COVID-19 }\end{array}$ & 13157
\end{tabular}


Aerosol and surface stability of HCoV-19 (SARS-CoV-2) compared to 11265 SARS-CoV-1

SARS-CoV-2 RNA concentrations in primary municipal sewage sludge 11152 as a leading indicator of COVID-19 outbreak dynamics

Hydroxychloroquine application is associated with a decreased mortality 10863 in critically ill patients with COVID-19

Hydroxychloroquine plus azithromycin: a potential interest in reducing 9386 in-hospital morbidity due to COVID-19 pneumonia (HI-ZY-COVID)?

Outcomes of hydroxychloroquine usage in United States veterans 8623

hospitalized

Hydroxychloroquine and azithromycin plus zinc vs hydroxychloroquine 6763

and azithromycin alone: outcomes in hospitalized COVID-19 patients

Correlation between universal BCG vaccination policy and reduced $\quad 6600$

mortality for COVID-19

Full lockdown policies in Western Europe countries have no evident $\quad 6124$

impacts on the COVID-19 epidemic

Suppression of COVID-19 outbreak in the municipality of Vo, Italy 5751

OpenSAFELY: factors associated with COVID-19-related hospital death 5417 in the linked electronic health records of 17 million adult NHS patients

A serological assay to detect SARS-CoV-2 seroconversion in humans 5373

Treatment Response to Hydroxychloroquine, Lopinavir/Ritonavir, and 4773

Antibiotics for Moderate COVID 19: A First Report on the

Pharmacological Outcomes from South Korea

Preliminary evidence from a multicenter prospective observational study 4747

of the safety and efficacy of chloroquine for the treatment of COVID-19

Saliva is more sensitive for SARS-CoV-2 detection in COVID-19 4573

patients than nasopharyngeal swabs

CoVID-19 in Japan: What could happen in the future? 4505

Effect of Dexamethasone in Hospitalized Patients with COVID-19: 4479

Preliminary Report

No evidence of clinical efficacy of hydroxychloroquine in patients 4343

hospitalized for COVID-19 infection with oxygen requirement: results of

a study using routinely collected data to emulate a target trial

Closed environments facilitate secondary transmission of coronavirus $\quad 4269$

disease 2019 (COVID-19)

Evolving Epidemiology and Impact of Non-pharmaceutical Interventions 4127

on the Outbreak of Coronavirus Disease 2019 in Wuhan, China 
Population-level COVID-19 mortality risk for non-elderly individuals

4053 overall and for non-elderly individuals without underlying diseases in pandemic epicenters Hydroxychloroquine in patients mainly with mild to moderate COVID- 3852 19: an open-label, randomized, controlled trial

Epidemiology and Transmission of COVID-19 in Shenzhen China: 3776 Analysis of 391 cases and 1,286 of their close contacts Aerosol and Surface Transmission Potential of SARS-CoV-2 3700

Estimation of seroprevalence of novel coronavirus disease (COVID-19) 3464 using preserved serum at an outpatient setting in Kobe, Japan: A crosssectional study.

Stability of SARS-CoV-2 in different environmental conditions

Effect of non-pharmaceutical interventions for containing the COVID-19 3361 outbreak in China

Relationship between the ABO Blood Group and the COVID-19

Susceptibility

The Novel Coronavirus, 2019-nCoV, is Highly Contagious and More 3103

Infectious Than Initially Estimated

Chloroquine diphosphate in two different dosages as adjunctive therapy

3028

of hospitalized patients with severe respiratory syndrome in the context of coronavirus (SARS-CoV-2) infection: Preliminary safety results of a randomized, double-blinded, phase IIb clinical trial (CloroCovid-19

Study)

Novel coronavirus 2019-nCoV: early estimation of epidemiological parameters and epidemic predictions

Neutralizing antibody responses to SARS-CoV-2 in a COVID-19 2909 recovered patient cohort and their implications

Humoral immune response and prolonged PCR positivity in a cohort of 2844 1343 SARS-CoV 2 patients in the New York City region

The infection fatality rate of COVID-19 inferred from seroprevalence 2789 data

Individual variation in susceptibility or exposure to SARS-CoV-2 lowers 2763 the herd immunity threshold

Clinical characteristics of 2019 novel coronavirus infection in China 2509

Differential Effects of Intervention Timing on COVID-19 Spread in the 2291 United States

EXCESS MORTALITY FROM COVID-19. WEEKLY EXCESS 2289 
JQD: DM 2(2022)

Politicization of Medical Preprints

\section{DEATH RATES BY AGE AND SEX FOR SWEDEN.}

The ABO blood group locus and a chromosome 3 gene cluster associate 2265 with SARS- CoV-2 respiratory failure in an Italian-Spanish $\mathrm{g}$ Favipiravir versus Arbidol for COVID-19: A Randomized Clinical Trial 2260

Hydroxychloroquine with or without azithromycin and in-hospital 2222 mortality or discharge in patients hospitalized for COVID-19 infection: a cohort study of 4,642 in-patients in France

Clinical presentation and virological assessment of hospitalized cases of 2221 coronavirus disease 2019 in a travel-associated transmission cluster Impact of non-pharmaceutical interventions against COVID-19 in 2209 Europe: a quasi-experimental study

Features of 16,749 hospitalised UK patients with COVID-19 using the 2202 ISARIC WHO Clinical Characterisation Protocol Vitamin D Insufficiency is Prevalent in Severe COVID-19 2128

Repeated seroprevalence of anti-SARS-CoV-2 IgG antibodies in a 2107 population-based sample from Geneva, Switzerland Estimating the generation interval for COVID-19 based on symptom 2081 onset data

ACE2 Expression in Kidney and Testis May Cause Kidney and Testis 2031 Damage After 2019-nCoV Infection

Estimation of SARS-CoV-2 mortality during the early stages of an epidemic: a modeling study in Hubei, China, and six regions in Europe Patient-derived mutations impact pathogenicity of SARS-CoV-2 1977

COVID-19 outbreak at a large homeless shelter in Boston: Implications for universal testing Factors associated with hospitalization and critical illness among 4,103 patients with COVID-19 disease in New York City Safety of hydroxychloroquine, alone and in combination with azithromycin, in light of rapid wide-spread use for COVID-19: a multinational, network cohort and self-controlled case series study A systematic review of antibody mediated immunity to coronaviruses: 1690 antibody kinetics, correlates of protection, and association The QT Interval in Patients with SARS-CoV-2 Infection Treated with 1658 Hydroxychloroquine/Azithromycin Neurological Manifestations of Hospitalized Patients with COVID-19 in 1634 Wuhan, China: a retrospective case series study 
Epidemiological Tools that Predict Partial Herd Immunity to SARS

1632

Coronavirus 2

Intervention strategies against COVID-19 and their estimated impact on 1617

Swedish healthcare capacity

Epidemiological and clinical features of the 2019 novel coronavirus 1571

outbreak in China

Intrafamilial Exposure to SARS-CoV-2 Induces Cellular Immune

1498

Response without Seroconversion

Presence of SARS-CoV-2 reactive T cells in COVID-19 patients and 1467

healthy donors

Antibody testing for COVID-19: A report from the National COVID 1442

Scientific Advisory Panel

Estimation of COVID-2019 burden and potential for international

dissemination of infection from Iran

Sentinel surveillance of SARS-CoV-2 in wastewater anticipates the 1368 occurrence of COVID-19 cases

A short therapeutic regimen based on hydroxychloroquine plus

azithromycin for the treatment of COVID-19 in patients with non-severe disease. A strategy associated with a reduction in hospital admissions and complications.

ICON (Ivermectin in COvid Nineteen) study: Use of Ivermectin is Associated with Lower Mortality in Hospitalized Patients with COVID19 Estimation of SARS-CoV-2 infection fatality rate by real-time antibody 1318 screening of blood donors

Cluster of COVID-19 in northern France: A retrospective closed cohort 1304 study

Pulmonary post-mortem findings in a large series of COVID-19 cases 1304 from Northern Italy

Effectiveness of isolation, testing, contact tracing and physical distancing 1214 on reducing transmission of SARS-CoV-2 in different settings Hydroxychloroquine and Azithromycin as a treatment of COVID-19: 1207 preliminary results of an open-label non-randomized clinical trial Collider bias undermines our understanding of COVID-19 disease risk 1195 and severity

Sex differences in immune responses to SARS-CoV-2 that underlie 1156 disease outcomes

Pre exposure Hydroxychloroquine use is associated with reduced COVID19 risk in healthcare workers - a Retrospective cohort Differential COVID-19-attributable mortality and BCG vaccine use in 
countries

Deep learning-based model for detecting 2019 novel coronavirus 1106 pneumonia on high-resolution computed tomography: a prospective study Viral Kinetics and Antibody Responses in Patients with COVID-19 1095

Antibody responses to SARS-CoV-2 in patients of novel coronavirus 1073 disease 2019

Using ILI surveillance to estimate state-specific case detection rates and 1010 forecast SARS-CoV-2 spread in the United States 


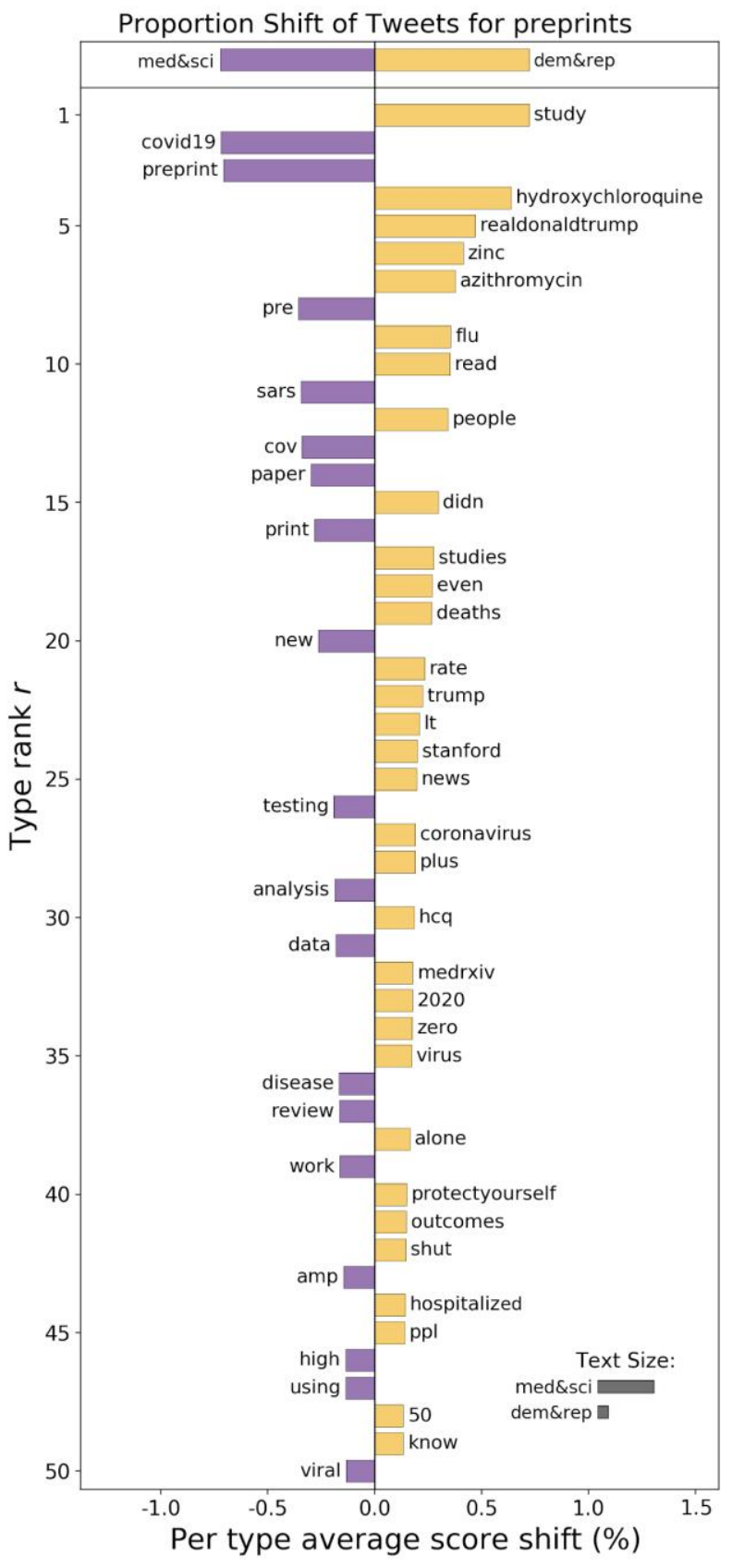

Figure A3. Word Shift Graph - Extended Version. 